\title{
Guest-editorial: 25 years of AI*IA
}

\author{
Matteo Baldoni ${ }^{\mathrm{a}, *}$, Paola Mello ${ }^{\mathrm{b}}$ and Fabrizio Riguzzi ${ }^{\mathrm{c}}$ \\ ${ }^{a}$ Dipartimento di Informatica, Università degli Studi di Torino, Torino, Italy \\ ${ }^{\mathrm{b}}$ Dipartimento di Informatica - Scienza e Ingegneria, Università di Bologna, Bologna, Italy \\ ${ }^{\mathrm{c}}$ Dipartimento di Matematica e Informatica, Università degli Studi di Ferrara, Ferrara, Italy
}

This issue reviews the recent work of the Italian community in Artificial Intelligence (AI). The study of AI has a long tradition in Italy, it can be dated back to the early Seventies and the research groups are numerous and their activities span a wide range of themes, from knowledge representation and automated reasoning, to machine learning, from multi-agent systems to robotics, and many others. A notable characteristic of Italian research is that it is evenly distributed throughout the country and the collaborations between universities and other institutions are quite tight. Such collaborations lead in 1988 to the foundation of the Associazione Italiana per l'Intelligenza Artificiale - Italian Association for Artificial Intelligence - (AI*IA), a non-profit scientific society devoted to the promotion of AI, aiming at increasing the public awareness of AI, encouraging and supporting the teaching of AI subjects and promoting research in the field. The founder and first chair of AI*IA was prof. Luigia Carlucci Aiello. Prof. Aiello, together with the other chairs and the association members, soon raised the association to the highest international levels and became the promoters of initiatives that provided both educational and scientific opportunities to many young researchers. One of the most peculiar characteristics of the association is that it includes several working groups, focused on specific topics, which organize yearly meetings of their members and which foster cross-fertilization among their members and members of other working groups. Indeed, the working groups are a driving force of the association.

This special issue is devoted to celebrate the 25th anniversary of the foundation of AI*IA. 25 years is

*Corresponding author: Matteo Baldoni, Dipartimento di Informatica, Università degli Studi di Torino, c.so Svizzera 185, I-10149 Torino, Italy. E-mail: matteo.baldoni@unito.it. a long period, especially for what concerns research. We are convinced that it is always important to take a break, look back and see what our community was and where it is going. This is particular important for young researchers, because the past and the present are fundamental to identify research directions. In this spirit, we asked the chair of each working group of AI*IA to write an article about the state of the art of the research in their area with a particular focus on the contribution of Italian researchers. The represented working groups are Machine Learning and Data Mining, Natural Language Processing, Artificial Intelligence for Cultural Heritage, Knowledge Representation and Automated Reasoning, Semantic Technologies, Robotics, and Agents and Multi-Agent Systems. All these papers show how AI is now a fundamental discipline for developing applications in many fields of computer and natural science, as well as social contexts such as smart cities, decision support systems for mobility and communication, energy, sustainability and health.

This special issue contains also a paper by the journalist and scientific popularizer Andrea Vico that last June interviewed the former and the actual chairs of AI*IA, Luigia Carlucci Aiello, Oliviero Stock, Pietro Torasso, Roberto Serra, Marco Gori, Marco Schaerf, and Paola Mello about their chairing stint, the challenges, the priorities and the achieved results.

The guest editors would like to thank all the people who contributed to this special issue: the authors and the reviewers, Oliviero Stock and the editorial board the Intelligenza Artificiale journal for the opportunity of realizing this special issue, and the Steering Committee of AI*IA.

Finally, we would like to say altogether:

Happy birthday AI*IA! 